\title{
Buprenorphine Provision by Early Career Family Physicians
}

\author{
Sebastian T. Tong, MD, MPH \\ Camille J. Hocbbeimer ${ }^{2}$ \\ Lars E. Peterson, MD, $\mathrm{PbD}^{3,4}$ \\ Alex H. Krist, MD, MPH ${ }^{1}$ \\ 'Department of Family Medicine and Popu- \\ lation Health, Virginia Commonwealth \\ University, Richmond, Virginia \\ ${ }^{2}$ Department of Biostatistics, Virginia \\ Commonwealth University, Richmond, \\ Virginia \\ ${ }^{3}$ Department of Family and Commu- \\ nity Medicine, University of Kentucky, \\ Lexington, Kentucky \\ ${ }^{4}$ American Board of Family Medicine, \\ Lexington, Kentucky
}

\begin{abstract}
Buprenorphine can be used in primary care to treat opioid use disorder, but many family physicians feel unprepared to care for patients with opioid addiction. We sought to describe preparedness to provide and current provision of buprenorphine treatment by early career family physicians using data from the 2016 National Family Medicine Graduate Survey. Of 1,979 respondents, 10.0\% reported preparedness to provide buprenorphine treatment, and $7.0 \%$ reported current buprenorphine provision. Residency preparation to provide buprenorphine treatment was most highly associated with current provision (odds ratio $=13.50 ; 95 \% \mathrm{Cl}, 7.59-24.03$ ). Efforts to increase buprenorphine training may alleviate the workforce shortage to treat opioid use disorder.
\end{abstract}

Ann Fam Med 2018;16:443-446. https://doi.org/10.1370/afm.2261.

\section{INTRODUCTION}

$\mathrm{D}$ eaths from drug overdose in the United States have increased from 16,651 in 2010 to more than 64,000 in 2016. ${ }^{1}$ An estimated 2.6 million Americans have opioid use disorder, but only $10.6 \%$ have received treatment. ${ }^{2}$ Untreated, this disorder has serious consequences, including legal problems and poor health outcomes. ${ }^{3}$

Buprenorphine, a medication approved by the Food and Drug Administration to treat opioid use disorder, reduces fatal overdoses ${ }^{4}$ and can be prescribed by primary care physicians who have obtained a prescribing waiver after completing 8 hours of training. Many have called for increasing access to buprenorphine. ${ }^{3}$ A recent study, however, reported that only $28.6 \%$ of family medicine residencies have a required addiction medicine curriculum, and only $31.2 \%$ had at least 1 graduate obtain a waiver to prescribe buprenorphine in the past year. ${ }^{5}$

Our study aimed to (1) describe preparedness to provide and current provision of buprenorphine by early career family physicians, and (2) determine associations between individual, residency, and practice characteristics with preparedness and current provision.

\section{METHODS}

Using a cross-sectional study design, we analyzed data collected from the 2016 National Family Medicine Graduate Survey administered to all American Board of Family Medicine (ABFM)-certified family physicians who completed their residency in 2013. The survey addressed whether the residency adequately prepared them to provide buprenorphine treatment and whether buprenorphine treatment was currently part of their practice. Survey details can be found elsewhere. ${ }^{6}$ We linked respondents to demographic characteristics collected by the ABFM and to their residency program. Residency program characteristics were obtained from the American Academy of Family Physicians Residency Directory ${ }^{7}$ and supplemented with the Teaching Health Center (THC) directory ${ }^{8}$ and the rural training track directory. ${ }^{9}$ Physicians who could not be matched to their residency program or who were not providing direct patient care were excluded.

\section{CORRESPONDING AUTHOR}

Sebastian T. Tong, MD, MPH 830 E Main St, Box 980101

Richmond, VA, 23298

stong@vcu.edu 
Table 1. Individual, Practice, and Residency Characteristics by Preparedness and Current Practice of Buprenorphine Treatment

\begin{tabular}{|c|c|c|c|c|c|c|}
\hline \multirow[b]{2}{*}{ Characteristics } & \multicolumn{3}{|c|}{ Prepared } & \multicolumn{3}{|c|}{ Practicing } \\
\hline & $\begin{array}{c}\text { Yes } \\
\text { No. (\%) }\end{array}$ & $\begin{array}{c}\text { No } \\
\text { No. (\%) }\end{array}$ & $\begin{array}{c}P \\
\text { Value }\end{array}$ & $\begin{array}{c}\text { Yes } \\
\text { No. (\%) }\end{array}$ & $\begin{array}{c}\text { No } \\
\text { No. (\%) }\end{array}$ & $\begin{array}{c}P \\
\text { Value }\end{array}$ \\
\hline \multicolumn{7}{|c|}{ Individual characteristics } \\
\hline \multicolumn{7}{|c|}{ Prepared to provide buprenorphine ${ }^{a}$} \\
\hline Yes & $\ldots$ & $\ldots$ & $\ldots$ & $63(45.7)$ & $135(7.3)$ & $<.01$ \\
\hline No & $\ldots$ & $\ldots$ & & $75(54.3)$ & $1,706(92.7)$ & \\
\hline \multicolumn{7}{|l|}{ Sex } \\
\hline Male & $81(40.9)$ & $785(44.1)$ & .95 & $67(48.5)$ & $799(43.4)$ & .10 \\
\hline Female & $117(59.1)$ & $996(55.9)$ & & $71(51.5)$ & $1,042(56.6)$ & \\
\hline Age & $36.4(4.4)$ & $35.9(4.4)$ & .16 & $36.1(4.5)$ & $35.9(4.4)$ & .38 \\
\hline \multicolumn{7}{|c|}{ Medical school type } \\
\hline Allopathic & $180(90.9)$ & $1,508(84.7)$ & .09 & $125(90.6)$ & $1,563(84.9)$ & .18 \\
\hline Osteopathic & $18(9.1)$ & $273(15.3)$ & & $13(9.4)$ & $278(15.1)$ & \\
\hline \multicolumn{7}{|l|}{ Loan program } \\
\hline Yes & 75 (37.9) & $609(34.2)$ & .94 & $53(38.4)$ & $631(34.3)$ & .49 \\
\hline No & $123(62.1)$ & $1,172(65.8)$ & & $85(61.6)$ & $1,210(65.7)$ & \\
\hline \multicolumn{7}{|l|}{ Research ${ }^{b}$} \\
\hline Yes & 38 (19.2) & $241(13.5)$ & .02 & $36(26.1)$ & $243(13.2)$ & $<.01$ \\
\hline No & $160(80.8)$ & $1,540(86.5)$ & & $102(73.9)$ & $1,598(86.8)$ & \\
\hline \multicolumn{7}{|l|}{ Faculty } \\
\hline Yes & $23(11.6)$ & 212 (11.9) & .98 & $23(16.7)$ & $212(11.5)$ & .17 \\
\hline No & $175(88.4)$ & $1,569(88.1)$ & & $115(83.3)$ & $1,629(88.5)$ & \\
\hline \multicolumn{7}{|c|}{ Residency characteristics } \\
\hline \multicolumn{7}{|c|}{$\mathrm{FQHC}$} \\
\hline Yes & $49(25.5)$ & $313(19.0)$ & .25 & $35(25.9)$ & $327(19.1)$ & .14 \\
\hline No & $143(74.5)$ & $1,339(81.0)$ & & $100(74.1)$ & $1,382(80.9)$ & \\
\hline \multicolumn{7}{|l|}{ THC funding } \\
\hline Yes & $9(4.6)$ & $66(3.7)$ & .70 & $11(8.0)$ & $64(3.5)$ & .06 \\
\hline \multirow[t]{2}{*}{ No } & $189(95.4)$ & $1,715(96.3)$ & & $127(92.0)$ & $1,777(96.5)$ & \\
\hline & & & & & & continues \\
\hline
\end{tabular}

We examined whether physician, residency, and practice characteristics were associated (1) with physicians being adequately prepared during residency to provide buprenorphine treatment and (2) with physicians currently providing buprenorphine treatment. A hierarchical model accounting for clustering within residencies was used to examine the association between these 2 outcomes and physician, residency, and practice characteristics. We considered physician and residency characteristics for both outcomes. For the outcome of current provision of buprenorphine, we additionally considered preparedness to provide buprenorphine and practice characteristics, but we excluded residency region because of collinearity with current practice region. Characteristics significantly associated with the outcome in a univariate model were entered into a multivariate model. Analyses were completed using SAS 9.4 (SAS Institute).
The Virginia Commonwealth University Institutional Review Board approved this study.

\section{RESULTS}

The response rate was $67.8 \%(2,069$ of 3,051$)$. Survey responders and nonresponders did not differ by age, sex, medical school type, or residency characteristics. After exclusions, our final sample size was 1,979 . Of respondents, $10.0 \%(n=198)$ reported being adequately trained during residency to provide buprenorphine treatment, and $7.0 \%(\mathrm{n}=138)$ reported providing buprenorphine treatment in their current practice. Of those currently providing buprenorphine, $45.6 \%(n=63)$ reported they were prepared to do so in residency. In addition, $31.8 \%$ of those who reported preparedness to provide buprenorphine treatment vs $4.2 \%$ those who did not are currently providing buprenorphine treatment. 
Table 1. Individual, Practice, and Residency Characteristics by Preparedness and Current Practice of Buprenorphine Treatment (continued)

\begin{tabular}{|c|c|c|c|c|c|c|}
\hline \multirow[b]{2}{*}{ Characteristics } & \multicolumn{3}{|c|}{ Prepared } & \multicolumn{3}{|c|}{ Practicing } \\
\hline & $\begin{array}{c}\text { Yes } \\
\text { No. (\%) }\end{array}$ & $\begin{array}{c}\text { No } \\
\text { No. (\%) }\end{array}$ & $\begin{array}{c}P \\
\text { Value }\end{array}$ & $\begin{array}{c}\text { Yes } \\
\text { No. (\%) }\end{array}$ & $\begin{array}{c}\text { No } \\
\text { No. (\%) }\end{array}$ & $\begin{array}{c}P \\
\text { Value }\end{array}$ \\
\hline \multicolumn{7}{|c|}{ Residency characteristics (continued) } \\
\hline \multicolumn{7}{|l|}{ Rural track } \\
\hline Yes & $19(9.6)$ & $142(8.0)$ & .88 & $9(6.5)$ & $152(8.3)$ & .45 \\
\hline No & $179(90.4)$ & $1,639(92.0)$ & & $129(93.5)$ & $1,689(91.7)$ & \\
\hline \multicolumn{7}{|l|}{ Residency region } \\
\hline Northeast & $65(32.8)$ & $278(15.6)$ & .01 & $33(23.9)$ & $310(16.8)$ & .01 \\
\hline South & $42(21.2)$ & $600(33.7)$ & & $35(25.4)$ & $607(33.0)$ & \\
\hline Midwest & $41(20.7)$ & $530(29.8)$ & & $26(18.8)$ & $545(29.6)$ & \\
\hline West & $50(25.3)$ & $373(20.9)$ & & $44(31.9)$ & $379(20.6)$ & \\
\hline Residents per class & $9.4(3.2)$ & $8.5(2.9)$ & .06 & $8.9(2.7)$ & $8.5(3.0)$ & .38 \\
\hline \multicolumn{7}{|c|}{ Practice characteristics } \\
\hline \multicolumn{7}{|c|}{$\mathrm{FQHC}$} \\
\hline Yes & $\ldots$ & $\ldots$ & $\ldots$ & $32(26.7)$ & $156(10.2)$ & .01 \\
\hline No & $\ldots$ & $\ldots$ & $\ldots$ & $88(73.3)$ & $1,374(89.8)$ & \\
\hline \multicolumn{7}{|l|}{ Rural } \\
\hline Yes & $\ldots$ & $\ldots$ & $\ldots$ & $3(2.5)$ & 68 (4.4) & .44 \\
\hline No & $\ldots$ & $\ldots$ & $\ldots$ & $117(97.5)$ & $1,462(95.6)$ & \\
\hline \multicolumn{7}{|l|}{ Site size } \\
\hline Solo practice & $\ldots$ & $\ldots$ & $\ldots$ & $8(6.7)$ & $50(3.3)$ & .10 \\
\hline 2-5 physicians & $\ldots$ & $\ldots$ & $\ldots$ & $34(28.3)$ & $575(37.6)$ & \\
\hline 6-20 physicians & $\ldots$ & $\ldots$ & $\ldots$ & $49(40.8)$ & $567(37.0)$ & \\
\hline$>20$ physicians & $\ldots$ & $\ldots$ & $\ldots$ & $29(24.2)$ & $338(22.1)$ & \\
\hline \multicolumn{7}{|l|}{ Site region } \\
\hline Northeast & $\ldots$ & $\ldots$ & $\ldots$ & $32(23.4)$ & $215(12.0)$ & $<.01$ \\
\hline South & $\ldots$ & $\ldots$ & $\ldots$ & $34(24.8)$ & $646(36.0)$ & \\
\hline Midwest & $\ldots$ & $\ldots$ & $\ldots$ & 19 (13.9) & $442(24.6)$ & \\
\hline West & $\ldots$ & $\ldots$ & $\ldots$ & $52(37.9)$ & $491(27.4)$ & \\
\hline
\end{tabular}

The relationships among physician, residency, and practice characteristics and each outcome are summarized in Table 1. In the multivariate model, those prepared to provide buprenorphine treatment were more likely to be engaged in research or practice-based research network (PBRN) activities and have trained in the Northeast or West as compared with the South or Midwest (Table 2). Those currently providing buprenorphine treatment were more likely to be prepared to provide this treatment, engaged in research or PBRN activities, work in a federally qualified health center (FQHC), and practice in the Northeast or West.

\section{DISCUSSION}

A minority of early career family physicians reported adequate preparation to provide buprenorphine treatment in residency, and even fewer provide it. Almost one-third of those prepared to provide buprenorphine in residency do so in current practice, suggesting that preparedness in residency may play a large role in current provision of buprenorphine treatment. Promoting buprenorphine treatment in residency training by addressing barriers identified by residency program directors $^{5}$ may help increase preparedness to provide buprenorphine.

Conversely, more than two-thirds of those prepared to provide buprenorphine in residency are not doing so in practice, suggesting there are logistical barriers to providing buprenorphine after graduation. ${ }^{3,10}$ Exploring ways to overcome these barriers may increase access to addiction services.

This study has several limitations. First, the crosssectional study design can determine associations only, not causation. Second, recall bias may exist as respondents' self-defined "preparedness" 3 years after 
Table 2. Adjusted, Significant Associations between Individual, Residency, and Current Practice Characteristics with Preparedness to Provide and Current Provision of Buprenorphine Treatment

\begin{tabular}{|c|c|c|}
\hline Characteristic & $\begin{array}{c}\text { Prepared to Provide } \\
\text { Buprenorphine } \\
\text { Treatment in Residency } \\
\text { OR }(95 \% \mathrm{Cl})\end{array}$ & $\begin{array}{l}\text { Currently Providing } \\
\text { Buprenorphine } \\
\text { Treatment } \\
\text { OR }(95 \% \mathrm{CI})\end{array}$ \\
\hline Prepared to provide buprenorphine ${ }^{a}$ & $\ldots$ & $13.50(7.59-24.03)$ \\
\hline Engaged in research ${ }^{b}$ & $1.69(1.05-2.72)$ & $2.17(1.28-3.67)$ \\
\hline \multicolumn{3}{|l|}{ Residency geographic region } \\
\hline Northeast ${ }^{c}$ & Reference & $\ldots$ \\
\hline Southe & $0.30(0.15-0.61)$ & $\ldots$ \\
\hline Midwest ${ }^{c}$ & $0.28(0.13-0.58)$ & $\ldots$ \\
\hline West ${ }^{c}$ & $0.52(0.24-1.11)$ & $\ldots$ \\
\hline \multicolumn{3}{|l|}{ Current practice geographic region } \\
\hline Northeast ${ }^{d}$ & $\ldots$ & Reference \\
\hline South $^{d}$ & $\cdots$ & $0.50(0.25-0.99)$ \\
\hline Midwest $^{d}$ & $\ldots$ & $0.37(0.16-0.84)$ \\
\hline West $^{d}$ & $\ldots$ & $0.87(0.45-1.69)$ \\
\hline Current practice in $\mathrm{FQHC}^{d}$ & $\ldots$ & $2.51(1.45-4.33)$ \\
\hline \multicolumn{3}{|l|}{ Current practice size } \\
\hline Solo practice ${ }^{d, e}$ & $\ldots$ & Reference \\
\hline 2-5 physicians d,e & $\ldots$ & $\ldots$ \\
\hline 6-20 physicians ${ }^{\text {d,e }}$ & $\ldots$ & $\ldots$ \\
\hline$>20$ physicians $^{\mathrm{d}, \mathrm{e}}$ & $\ldots$ & $\cdots$ \\
\hline \multicolumn{3}{|c|}{$\mathrm{FQHC}=$ federally qualified health center; $\mathrm{OR}=$ odds ratio. } \\
\hline $\begin{array}{l}\text { a Preparedness is self-reported as prepared } \\
\text { b Research is self-reported in the survey as } \\
\text { activities. } \\
\text { ' Not included in currently providing treat } \\
\text { d Not included in prepared to provide ana } \\
\text { e Not significant in currently providing tre }\end{array}$ & $\begin{array}{l}\text { residency to provide buprenorphin } \\
\text { gagement in research or practice-b } \\
\text { ent analysis. } \\
\text { is. } \\
\text { nent multivariate analysis. }\end{array}$ & $\begin{array}{l}\text { treatment. } \\
\text { sed research network }\end{array}$ \\
\hline
\end{tabular}

To read or post commentaries in response to this article, see it online at http://www.AnnFamMed.orgl content/16/5/443.

Submitted January 13, 2018; submitted, revised, March 26, 2018; accepted April 6, 2018.

Key words: primary health care; buprenorphine; opiate substitution treatment; health manpower

Previous presentations: Society of Teachers of Family Medicine Annual Spring Conference, May 2018, Washington, D.C.; North American Primary Care Research Group Annual Meeting, November 2017, Montréal, Québec, Canada.

Acknowledgments: Bo Fang, PhD, extracted the data from the ABFM National Family Medicine Graduate Survey and provided nonresponder characteristics.

\section{References}

1. National Institute on Drug Abuse. Overdose death rates. https://www. drugabuse.gov/related-topics/trendsstatistics/overdose-death-rates. Published 2017. Accessed Jan 13, 2018.

2. Center for Behavioral Health Statistics and Quality. Key Substance Use and Mental Health Indicators in the United States: Results from the 2015 National Survey on Drug Use and Health. https:// www.samhsa.gov/data/sites/default/files/ NSDUH-FFR1-2015/NSDUH-FFR1-2015/ NSDUH-FFR1-2015.pdf. Published Sep 2016. Accessed Jan 13, 2018.

residency training. Third, limited data on residency curricula meant that we could not assess associations between curricula and preparedness. Fourth, despite a decent sample size, the percentage in each outcome was small, and our analysis may be underpowered. Fifth, residents who are interested in treating opioid use disorder may self-select into residencies that adequately prepare them to provide buprenorphine. Finally, demand for buprenorphine treatment was not considered in the model.

Few early career family physicians reported being prepared to provide or offer current provision of buprenorphine treatment, and even most of those prepared to offer it do not include it in their practice. Efforts to increase residency training in buprenorphine treatment, engage practices through PBRNs, and support FQHCs may increase buprenorphine provision by family physicians. In addition to addressing the opioid epidemic, these efforts would improve continuity of care, legitimize opioid use disorder as a chronic disease, and destigmatize its treatment.
3. Volkow ND, Frieden TR, Hyde PS, Cha SS. Medication-assisted therapies-tackling the opioid-overdose epidemic. N Engl J Med. 2014; 370(22):2063-6.

4. Schwartz RP, Gryczynski J, O’Grady KE, et al. Opioid agonist treatments and heroin overdose deaths in Baltimore, Maryland, 19952009. Am J Public Health. 2013;103(5):917-22.

5. Tong S, Sabo R, Aycock R, et al. Assessment of addiction medicine training in family medicine residency programs: a CERA study. Fam Med. 2017;49(7):537-543.

6. Weidner A, Chen F, Peterson LE. Developing the National Family Medicine Graduate Survey. J Grad Med Educ. 2017;9(5):570-3.

7. American Academy of Family Physicians. Residency directory. https://nf.aafp.org/Directories/Residency/Search. Published 2015. Accessed Nov 6, 2016.

8. Health Resources $\&$ Services Administration. Teaching health center GME 2014 grant awards. http://www.hrsa.gov/about/ news/2014tables/teachinghealthcenters/. Published 2014. Updated Mar 2016. Accessed Nov 6, 2016.

9. RTT Collaborative. Directory of rural programs. https:// rttcollaborative.net/rural-programs/. Accessed Nov 6, 2016.

10. Hutchinson E, Catlin M, Andrilla CHA, Baldwin L-M, Rosenblatt RA. Barriers to primary care physicians prescribing buprenorphine. Ann Fam Med. 2014;12(2):128-33. 